\title{
Design and Simulation of Closed-Loop Control System for Screw Oil Press
}

\author{
Z.G. Hu \\ Department of Mechanical Engineering \\ Wuhan Polytechnic University \\ China
}

\author{
Y.L. Chen \\ Department of Mechanical Engineering \\ Wuhan Polytechnic University \\ China
}

\begin{abstract}
The traditional screw press has many disadvantages, such as the lower level of automation, the existing unstable control system and the longer response time. Through the research on the principle of pressed oil, mechanical structure and the automatic control procedure of the screw press, a set of automatic closed-loop control system of the screw press has been designed, which is based on Programmable Logic Controller (PLC) control and Proportion - Integration Differentiation (PID) control strategy. For this control system a simulation model is established using Simulink, and the simulation experiment and analysis are also presented. It is shown that this system could greatly improve the level of automation of the screw press and provides certain reference to the design of intelligent control system used for large oil press.
\end{abstract}

Keywords-screw press; closed-loop control; PLC; PID controller; simulink simulation

\section{INTRODUCTION}

China is a popular country. In recent years, as people's living standards continue to improve, the demand of edible cooking oil becomes huger. Nowadays, there are two main types of vegetable oils extraction method: leaching method and mechanical crushing method. The hydraulic and the screw oil press are two main types of mechanical crushing equipment, and screw press is the predominant equipment of mechanical press. According to statistics [1], the social ownership of screw oil press in China has reached to1.3 million units. Each year, more than 45 percent of vegetable oils are extracted through the screw press, which occupy an important position in China's pressed oil industry. Thus the screw press is, as it were, a kind of oil processing machinery that conforms to the situation of our country. However, the screw press has a lower level of automatic control in the process of extracting oil. Due to workers' improper operation, it is often the case to cause serious waste of energy and resources [2-3]. Therefore, how to improve the automation level of the screw press is a problem to be solved.

For the traditional screw press, it can't know clearly how long it takes for a batch of oil materials to be squeezed out. Therefore, in order to avoid energy waste caused by the operation of workers, one should clarify the actuation sequence and the control process when the screw press operates in pressed oil procedure. Obviously, open-loop control system without the information feedback cannot meet the control and actual operating requirements. So the closed-loop control system based on PLC can be introduced to realize the automation control with the PID controller [4-6]. Moreover, the use of Simulink to establish simulation model of a control system and to test its performance can facilitate and improve the design of a control system. Earlier, Chen Yong [7] used relay-contactor to design an open-loop control system for the hydraulic oil press. In order to obtain some good dynamic performance, Qi Wen-Zhe [8] etc. used PID controller to the control system, and optimized PID control parameters via using Simulink simulation test. In this article, PLC control is adopted to develop a closed-loop control system for a screw press. The transfer function model of the controlled object and the PID controller is first established, and then Simulink simulation is conducted to make clear dynamic properties of the control system under different control parameters. Meanwhile, optimal control parameters are to be chosen.

\section{CLOSED-LOOP CONTROL SYSTEM DESIGN FOR A SCREW PRESS}

\section{A. Press Principle of Screw Press}

Screw pressed oil principle is as follow: the oil materials in the hopper are pushed into the pressing chamber using the feed screw. The squeezing screw turns to make the material full of the pressing chamber, and squeeze them in the chamber. The oil embryo materials get a great frictional resistance between the chamber and the squeezing screw under the high pressure condition of pressing chamber, which can cause the friction and form the relative motion between material particles. Every embryo material particle inside the chamber does not move in a constant velocity and the same move direction. The heat generated by the friction can satisfy the requirement for the quantity of heat that is necessary to the pressed oil process. As the pressure increases gradually, a large number of oil is discharged, forms oil fluids and flows out from a row of aperture on the pressing chamber. The remainder of oil materials will eventually be solidified, and becomes cake formation. Thus it ends pressed oil process [9].

\section{B. Press System of Screw Press}

At present, the pressure system of traditional screw presses widely adopts single screw press. The feeding speed and feeding rate of the materials are controlled by the feeding motor using manual control. The feeding control 
system is usually open-loop. The total squeezing time is set up in the system before the squeezing. While its manufacturing cost is low, the configuration of the oil press is simple and its maintenance is convenient, but the manual regulation is of low accuracy. When the pressure fluctuation occurs in the pressing chamber, the operator can't control the feeding motor speed accurately. So it is difficult to ensure uniform feeding rate and feed speed, leading to squeezing pressure uncontrollable in the chamber and the pressing quality unsteady [10].Modern advanced screw press system has used a closed-loop control technique, which controls the feeding rate and feed speed in pressing chamber, and has equipped with the forced feeding device. It ensures the feed speed controllable in order to achieve the purpose of controlling the pressure of pressing chamber.

The screw press structure is mainly composed of hopper, feed screw, feed motor, squeezer, pressing chamber, spindle motor, feed screw, pressure sensors and other components, as shown in Figure 1.

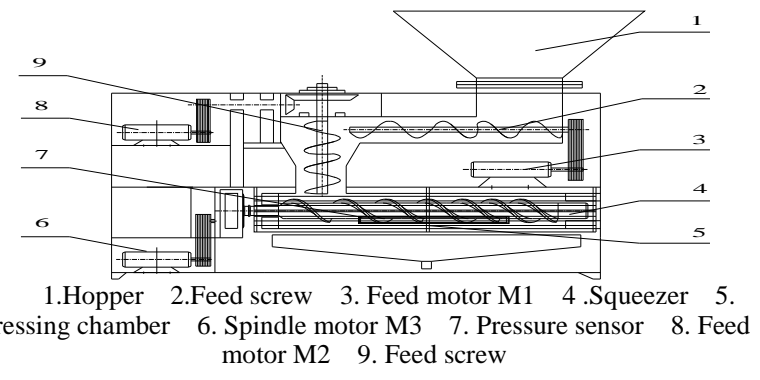

FIGURE I. SCREW PRESS STRUCTURE.

\section{Control System of Screw Press}

To prevent the grease protein denaturation and the quality of vegetable oil deterioration due to high temperature of the pressed oil, it is necessary to control the pressure of the chamber. In the process of screw pressing, the feed speed into the chamber is the key control parameter. The squeezing feed speed directly affects the squeezing pressure in the chamber. The high chamber pressure directly causes the chamber temperature increasing inside the chamber, and the oil quality cannot be guaranteed. The small chamber pressure results in insufficient squeezing and serious oil materials waste, and affect the working efficiency and yield efficiency. Based on the above analysis, a set of closed-loop control system for the screw press based on PLC is proposed.

1) Control system configuration: The control system is composed of PLC controller, inverter, feeding control system, pressure sensor, A/D module, etc, as shown in figure 2.

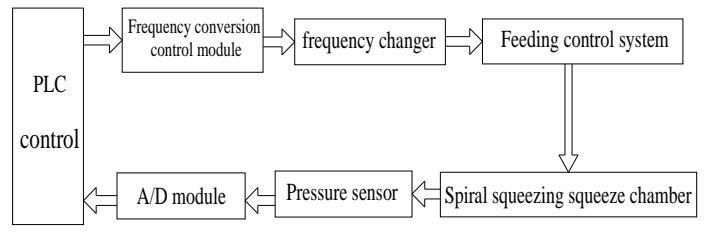

FIGURE II. BLOCK DIAGRAM OF THE CONTROL SYSTEM.
The control system of screw press takes the feeding motor as the controlled object and feedback element is a pressure sensor installed inside the chamber. The system input is the squeezing pressure inside the chamber and the output is the feeding motor speed.

2) Control strategy and algorithm: In the simulating condition and real digital control, traditional PID control has been widely used. PID control has advantage of convenient adjustment, good adaptability and stability, etc. Three control parameters of PID controller directly affect the effects of PID control. Matlab simulation of the PID controller can optimally set three control parameters in order to make PID to achieve the expected control effect [11-13].

3) System control model: PLC can control the turning speed of the feeding motor to adjust the feed speed. Pressure sensors measure the pressure inside the chamber. Its signals give feedback to the feeding motor to achieve independently closed-loop control for the pressure of the squeezing chamber using PID control algorithm. The control block diagram is shown in figure 3.

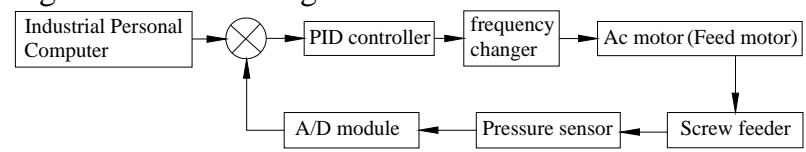

FIGURE III. CONTROL BLOCK DIAGRAM.

4) System control process: Mitsubishi FX2N - $48 \mathrm{MR}$ is chosen as PLC in the control system. The pressure sensor inside the chamber transmits squeezing pressure values via analog signals, which are transformed into digital signals through A/D module to the PID controller. When the squeezing pressure inside the chamber is less than the setting value, the feeding motor speed is sped up, which increases squeezing forces within the chamber. When the squeezing pressure detected in the chamber is greater than the setting value, the feeding motor speed is decreased, which reduces squeezing forces within the chamber. Time used for the detection, comparison and control is of very short (less than $20 \mathrm{~ms}$ ), which can realize real-time closed-loop control of the pressed oil process. The control process is shown in figure 4 .

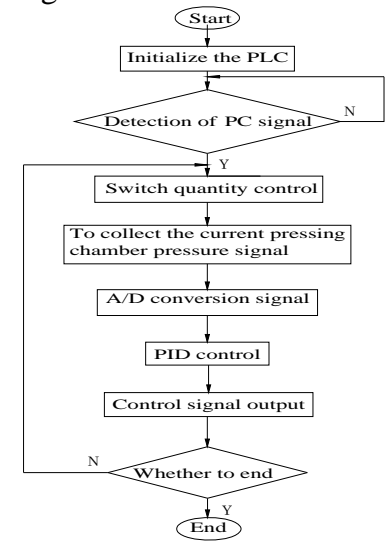

FIGURE IV. FLOW CHART OF CONTROL ALGORITHM. 


\section{CLOSED-LOOP CONTROL SYSTEM SIMULATION OF SCREW PRESS}

\section{A. Establishing Mathematical Model}

The control system uses a continuous control system with the unit feedback. The system control process as shown in figure 5 .

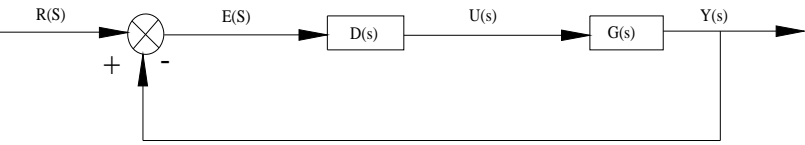

FIGURE V. CONTINUOUS CONTROL SYSTEM BLOCK DIAGRAM.

The transfer function of the PID controller[14] has the following form:

$$
D(s)=K_{p}+K_{i}+K_{d}
$$

Where $\mathrm{Kp}$ is proportional coefficient, $\mathrm{Ki}$ integral coefficient and Kd differential coefficient.

The transfer function for the controlled object and photosensitive sensor (motor) has the following form[15]:

$$
G(s)=\frac{5}{s^{2}+3 s+3}
$$

Simulink simulation[16] was carried out through the computer technology to adjust the PID controller parameter values, which makes the dynamic performance to achieve the best.

\section{B. Set Up Simulink Simulation Model}

Modeling purpose is to transform mathematical model into the model that computers are able to identify. According to the continuous control block diagram as shown in figure 5 and the control rules of PID controller, using Simulink toolbox, the control system simulation model is established as shown in figure 6 .

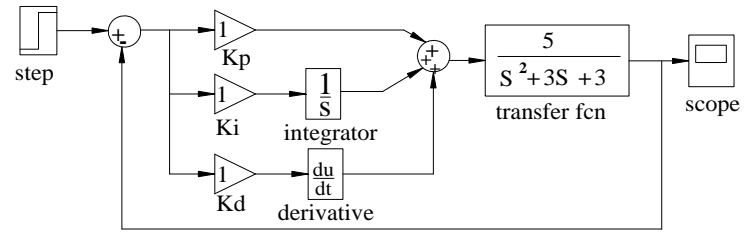

FIGURE VI. THE SIMULATION MODEL.

\section{Simulation Experimental Data}

After the construction of the Simulink simulation model, single variable control method is used to determine optimal control parameters of PID controller. The so-called single variable control method is to only let a variable change while ensure that the other two variables keep constant in order to find the optimal value of the variables. repeatedly to change the value of the input quantity, to run the Simulink simulation program, one can observe the response speed of system through the oscilloscope observation system. Through adjusting the three control parameters for many times, the dynamic performance of the system could achieve optimal in terms of the transient performance index, the maximum overshoot, the adjusting time and steady-state error. Table 1 is some simulation test data with the single variable control method.

TABLE I. PID SIMULATION EXPERIMENTAL DATA.

\begin{tabular}{cccccc}
\hline $\boldsymbol{K}_{\boldsymbol{p}}$ & $\boldsymbol{K}_{\boldsymbol{i}}$ & $\boldsymbol{K}_{\boldsymbol{d}}$ & $\boldsymbol{M m a x}$ & $\boldsymbol{T} \boldsymbol{y}$ & $\boldsymbol{y}(\boldsymbol{t})$ \\
\hline 0.05 & 0 & 0 & - & 5.0 & 0.143 \\
0.25 & 0 & 0 & - & 4.5 & 0.455 \\
0.45 & 0 & 0 & - & 5.0 & 0.600 \\
0.65 & 0 & 0 & - & 5.5 & 0.684 \\
0.85 & 0 & 0 & - & 6.0 & 0.739 \\
1.0 & 0 & 0 & - & 6.5 & 0.769 \\
0.05 & 0.1 & 0 & - & 9.5 & 0.999 \\
0.05 & 0.15 & 0 & - & 6.0 & 0.999 \\
0.05 & 0.2 & 0 & - & 6.0 & 0.999 \\
0.05 & 0.25 & 0 & 1.112 & 9.0 & 1.0 \\
0.05 & 0.3 & 0 & 1.198 & 9.5 & 1.0 \\
0.05 & 0.3 & 0.1 & 1.356 & 12.5 & 1.0 \\
0.05 & 0.3 & 0.35 & 1.389 & 17.5 & 1.0 \\
\hline Several & groups of representative data & & \\
\hline
\end{tabular}

Several groups of representative data are chosen from the above table, and the system responses are shown as in figure 7 .
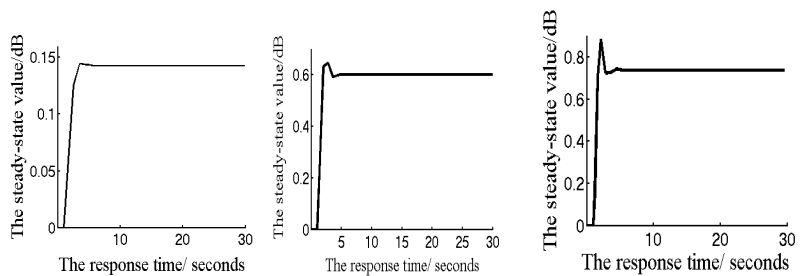

(a) $K_{p}=0.05, K_{i}=K_{d}=0$

(b) $K_{p}=0.45, K_{i}=K_{d}=0$
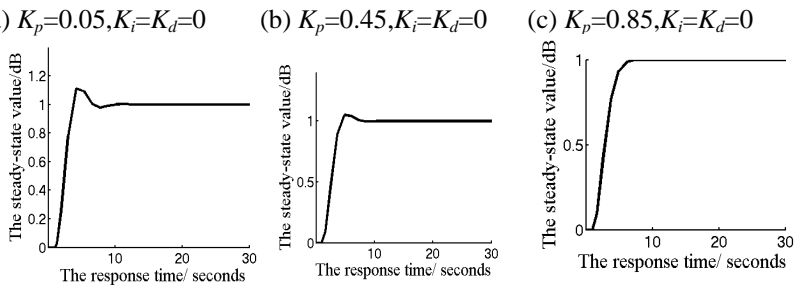

(d) $K_{p}=0.05, K_{i}=0.25, K_{d}=0$ (e) $K_{p}=0.05, K_{i}=0.2, K_{d}=0$ (f) $K_{p}=0.05, K_{i}=0.15, K_{d}=0$ FIGURE VII.

CONTROL SYSTEM SIMULATION RESULTS.

\section{Simulation Results}

By simulation test data and simulation response results, the following conclusions can be drew:

(1) Improving the proportion parameters Kp can speed up system responses and reduce the dynamic error;

(2) The differential control increases the amount of overshoot of the system, which should be reduced appropriately.

(3) When the proportion parameters $\mathrm{Kp}$ is fixed, appropriately increasing the coefficient of integral control can eliminate the steady-state error to some extent;

(4) It can be seen from the simulation results in the figures that, when $\mathrm{Kp}=0.05, \mathrm{Ki}=0.15, \mathrm{Kd}=0$, the system can gain good control effect, fast response speed and no 
overshoot.

\section{CONCLUSION}

For screw press some typical working conditions in batch production are analyzed. The working characteristics and actual work requirements are presented according to the actual design requirements. With the design requirements, the control system and simulation test platform for the design are established. The design method of reset instruction sequence control system and Simulink toolbox are used to complete the design and simulation of the control system. Through the simulating experiments, the screw press control system is designed via the simulation analysis. The simulation experiments show that the design of the closed-loop automatic control system can improve the automation level of the screw press. Some existing problems of the control system, such as the long system response times, are solved. However, as the PID controller parameters are obtained by a single variable testing method, it cannot ensure the accurate control parameters. How to get the optimal PID control parameters by calculating also remains to be further researched.

\section{REFERENCE}

[1] Wu Guoxian. The screw press industry in our country situation analysis [J]. Agricultural machinery testing and promotion. 1998 (5) : 10 11.

[2] Liu Yi. Sun Benzhu. Zhao. Ren Feng. Screw press industry present situation and the quality analysis [J]. Journal of agricultural science and technology and equipment. 2010 (12) : 71 72.

[3] Gao Jing. Screw press industry quality and countermeasures [J]. Journal of agricultural science and technology and equipment. 2010 (4) : 116 118.

[4] Tan YongHong. Introductions of PID Controllers and Intelligent Control [J]. Journal of Hefei University of Technology, 2002, (5) : 109 117.

[5] Xiao-dan Li. A fuzzy PID controller design research [D]. Tianjin: tianjin university, 2005.

[6] YangZhi. Zhu Haifeng. Huang YiHua. The PID controller design and parameter setting method of review [J]. Journal of chemical industry automation and instrumentation. 2005, 32 (5):1 7.

[7] Chen yong. The design of the hydraulic oil mill automatic control system [J]. Journal of agricultural mechanization research. 2008 (2) : $194 \sim 195$.

[8] QiWenZhe. yu-cheng wang. Control system design based on Matlab and simulation study [J]. Journal of laboratory science. 2008 (8) : 73 75.

[9] Zheng Xiao. Theory and experimental study on the oil press [D]. Wuhan: wuhan university of technology, 2005.

[10] Da-Chuan Liu. Jin-bo Liu. Zhang Lin. Xiao-qin Liu. Liu Ye. Automatic pressing chamber pressure control screw press: China, 201320165110.4 [P]. 2013-09-04.

[11] Xu Ping. Li Geng. Wang Kai. An improved PID parameter setting method [J]. Journal of modern electronic technology. 2012 (5) : $150 \sim 156$.

[12] OuYangHuiBin.YangWu jiao. Simulation of PID parameter setting method [J]. Computer simulation. 2007 (7) : 323 325.

[13] Rui-Ling $\mathrm{Fu}$. Li-Qin Le. Setting the PID parameter based on MATLAB/Simulink [J]. Industrial control computer. 2013, 26 (8) : 75 76.

[14] Zhi-qian Yu. Based on kingview and PLC and frequency converter in the design of constant pressure water supply control system [D]. Shandong, shandong university, 2008.

[15] kingview intermediate training tutorial. Beijing and control technology co., LTD.

[16] Zheng-lin Wang. Sheng-kai Wang. MATLAB/Simulink simulation and control system [M]. Beijing. Electronic industry press, 2013. 45 100. 